\title{
Effect of Polymer Molecular Weight on the Bone Biological Activity of Biodegradable Polymer/Calcium Phosphate Cement Composites
}

\author{
Esther W.H. Bodde, M.D., Ph.D., Wouter J.E.M. Habraken, Ph.D., ${ }^{1}$ Antonios G. Mikos, Ph.D., \\ Paul H.M. Spauwen, M.D., Ph.D., ${ }^{3}$ and John A. Jansen, D.D.S., Ph.D.
}

Previous studies demonstrated that the addition of biodegradable polymer microparticles to calcium phosphate $(\mathrm{CaP})$ cement improves the cement's degradative behavior without affecting its handling characteristics, especially its injectability and moldability. We investigated the influence of molecular weight of polymeric microparticles included in $\mathrm{CaP}$ cement on implant degradation and bone formation in critical-sized defects. Forty rats received cranial defects filled with formulations of CaP cement and poly(DL-lactic-co-glycolic acid) (PLGA) microparticles. Microparticles consisted of 100\% high- (HMW) or low-molecular-weight (LMW) PLGA or mixtures of these (25\%, $50 \%$, or $75 \%$ ). Implantation time was 12 weeks. Porosity measurements showed that the $100 \%$ HMW group was significantly less porous than the other groups. Histology and histomorphometry revealed significantly greater implant degradation in the $100 \%$ LMW group. Defect bridging was mainly seen in the $75 \%$ and $100 \%$ LMW groups, with the highest amount of bone in the 100\% LMW formulation. These results suggest that LMW PLGA microparticles are associated with better bone formation than HMW PLGA, which is most likely explained by the greater degradation of LMW PLGA microparticles. In conclusion, CaP cement composites with high percentages of LMW PLGA microparticles show good bone transductive behavior, with complete defect bridging. The 100\% LMW group turned out to be the best formulation.

\section{Introduction}

C alcium phosphate (CaP) cements can be good bone graft substitutes to fill bone defects; obtain fracture stability; or fixate prostheses in dental, orthopedic, or reconstructive surgery applications because of their biocompatibility and capability for osteoconduction. ${ }^{1-4}$ Before setting, the paste can be molded in the desired shape, or it can be directly injected and harden in situ. A disadvantage of the cement, however, is its poor biomechanical and biodegradation behavior..$^{5-8}$

The degradation rate of cement can be improved by increasing its porosity and thereby increasing the specific surface area and the possibility for cells to enter the scaffold. ${ }^{9}$ There are several ways to create macropores in cement, but the injectable and moldable properties are not always maintained. A technique to create macropores without losing the handling properties is the inclusion of biodegradable polymeric microparticles. ${ }^{10-12}$ The microparticles will initially stabilize the cement but will also degrade over time, leaving macropores behind. The use of poly(DL-lactic- co-glycolic acid) (PLGA) as source for porogens is a favorable possibility, because these microparticles can be used as a delivery vehicle for growth factors and to further enhance the bone regenerative effect of the $\mathrm{CaP}$ cement. ${ }^{13,14}$ It must be emphasized that the inclusion of PLGA microparticles is not intended to increase the mechanical properties of the $\mathrm{CaP}$ cement. This must be achieved by the degradation of the PLGA micropartcles and the subsequent ingrowth of bone. In a previous study, $\mathrm{CaP}$ cement with PLGA microparticle composites were implanted subcutaneously and in noncritical-sized ( $3.5 \mathrm{~mm}$ in diameter) cranial defects in rats. The study showed that high-molecular-weight (HMW) PLGA degraded in vivo and that the pores formed subsequently allowed ingrowth of soft and hard tissue throughout the specimens after 12 weeks of implantation. ${ }^{12}$ For the further evaluation of the regeneration capacity of the composite, the material was recently investigated in critical-sized $(8 \mathrm{~mm}$ in diameter) cranial defects in rats. ${ }^{15}$ In these implants, ingrowth of bone and soft tissue was seen only superficially and not in the center, even after 12 weeks of implantation. Evidently, the degradation of the microparticles in the

\footnotetext{
${ }^{1}$ Department of Periodontology and Biomaterials, Radboud University Nijmegen Medical Center, Nijmegen, The Netherlands.

${ }^{2}$ Department of Bioengineering, Rice University, Houston, Texas.

${ }^{3}$ Department of Plastic and Reconstructive Surgery, Radboud University Nijmegen Medical Center, Nijmegen, The Netherlands.
} 
"critical-sized implants" differed from that in the previous "non-critical-sized implants". Therefore, to optimize the composite material, the degradation of the microparticles should be increased.

One option to vary the degradation pattern of polymeric microspheres is to change the molecular weight of PLGA (e.g., low-molecular-weight (LMW) PLGA degrades faster than high HMW PLGA. ${ }^{16}$ A mixture of LMW and HMW PLGA might be the optimal combination for initial stabilization and implant degradation over time, leading to greater bone formation in critical-sized defects than with a composite of $\mathrm{CaP}$ cement and solely HMW PLGA.

Based on the considerations mentioned above, the purpose of the present study was to determine the influence of different molecular weights of PLGA microspheres on implant degradation and bone ingrowth in $\mathrm{CaP}$ cement implants placed in critical-sized bone defects in rats. We hypothesized that a high percentage of LMW PLGA microparticles in the $\mathrm{CaP}$ cement would result in an better osseotransductive behavior of the cement than the inclusion of HMW PLGA microparticles.

\section{Materials and Methods}

\section{Materials}

The CaP cement (Calcibon, Biomet Merck, Darmstadt, Germany) consisted of $61 \%$ alpha-tricalcium phosphate, $26 \%$ dicalcium phosphate dihydrate, $10 \%$ calcium carbonate, and $3 \%$ precipitated hydroxy apatite. The cement powder was sterilized using gamma radiation with $25 \mathrm{kGy}$ (Isotron B.V., Ede, The Netherlands). The cement liquid applied was a filter-sterilized $(0.2-\mu \mathrm{m}$ filter) $1 \%$ aqueous solution of sodium phosphate. Poly (DL-lactic-co-glycolic acid) (PLGA) (Purasorb, Purac, Gorinchem, The Netherlands) with a lactic to glycolic acid ratio of 50:50 and an average molecular weight $\left(\mathrm{M}_{\mathrm{w}}\right)$ of $54.21 \pm 1.34 \mathrm{kDa}(\mathrm{HMW})$ and $4.55 \pm 0.03 \mathrm{kDa}(\mathrm{LMW})$ was used for the microparticle preparation. The number average molecular weights and polydispersity index of the polymers were $34.73 \pm 0.38 \mathrm{kDa}$ and $1.56 \pm 0.03$, respectively, for HMW and $2.84 \pm 0.003 \mathrm{kDa}$ and $1.60 \pm 0.01$, respectively, for LMW.

\section{Preparation of PLGA microparticles}

To prepare PLGA microparticles, a double-emulsion solvent-extraction technique ([water in oil] in water) was used. ${ }^{11,13,17-19}$ HMW PLGA microparticles were produced by injecting $500 \mu \mathrm{L}$ of distilled water into a tube containing a solution of $1.0 \mathrm{~g}$ of HMW PLGA in $4 \mathrm{~mL}$ of dichloromethane. The mixture was emulsified for $60 \mathrm{~s}$ on a vortexer. Accordingly, LMW PLGA microparticles were produced by adding $500 \mu \mathrm{L}$ of distilled water to $1400 \mathrm{mg}$ of LMW PLGA in $2 \mathrm{~mL}$ of dichloromethane to obtain microparticles of similar sizes as the HMW PLGA. Then $6 \mathrm{~mL}$ of $0.3 \%$ aqueous poly (vinyl alcohol) (PVA, Acros Organics, Geel, Belgium) solution was added and emulsified for another $60 \mathrm{~s}$ to produce the second emulsion. This mixture was added to $394 \mathrm{~mL}$ of $0.3 \%$ PVA solution and $400 \mathrm{~mL}$ of $2 \%$ isopropyl alcohol solution and was stirred for $1 \mathrm{~h}$. The evaporation of the solvent resulted in precipitation of the dissolved polymer, and microparticles were subsequently formed. The microparticles were allowed to settle for $15 \mathrm{~min}$, and the solution was decanted. Then the microparticles were collected through centrifugation at $1500 \mathrm{rpm}$ for $5 \mathrm{~min}$, lyophilized to dryness, and stored under argon at $-20^{\circ} \mathrm{C}$ until use. The size distribution of the PLGA microparticles was determined using image analysis (Leica Qwin, Leica Microsystems, Wetzlar, Germany). Scanning electron micrography (SEM) images of the microparticles were made at the Microscopic Imaging Center of the Nijmegen Center for Molecular Life Sciences (Nijmegen, The Netherlands).

\section{Preparation of $\mathrm{CaP}$ cement implants}

The PLGA microparticles were mixed with the CaP cement powder in a proportion of $20 \mathrm{wt} \%$, because a previous study indicated this as the best ratio. ${ }^{11}$ Different ratios of HMW to LMW PLGA microparticles were used, corresponding to the different groups: I. 100\% HMW PLGA (100HMW); II. 25\%/ 75\% LMW/HMW PLGA (25LMW); III. 50\%/50\% LMW/ HMW PLGA (50LMW); IV. 75\%/25\% LMW/HMW PLGA (75LMW) and V. 100\% LMW PLGA (100LMW). The cement was created by adding $1 \mathrm{wt} \%$ sodium phosphate to the PLGA/CaP powder mixture using a 2-mL syringe (BD Plastipak, Becton Dickinson S.A., Madrid, Spain) with a closed tip and shaking these components for $15 \mathrm{~s}$ using a mixing apparatus (Silamat, Vivadent, Schaan, Liechtenstein). The ratio of liquid to powder ranged from 0.35 for the 100\% HMW to 0.5 for the $100 \%$ LMW groups. The cement was immediately injected into Teflon molds with a standardized shape $(7.8 \mathrm{~mm} \times 1.8 \mathrm{~mm})$. After setting at room temperature overnight, the implants were removed from the molds. SEM images (Jeol JSM 633OF) were made of the composites at the Department of Natural Sciences of the Radboud University (Nijmegen, The Netherlands).

\section{Porosity measurements}

The macroporosity and total porosity were determined for the different implant formulations. PLGA-CaP cement samples $(n=6)$ were placed in a furnace at $650^{\circ} \mathrm{C}$ for $2 \mathrm{~h}$, to burn out the polymer. Then, by weighing the implants, the total porosity and macroporosity could be calculated using equations 1 and 2 as described in the study of Habraken et al. ${ }^{11}$

$$
\begin{array}{rlr}
\varepsilon_{\text {tot }} & =\left(1-\frac{m_{\text {burnt }}}{V^{*} \rho_{\text {HAP }}}\right) * 100 \% & \text { Equation } 1 \\
\varepsilon_{\text {macro }} & =\left(1-\frac{m_{\text {burnt }}}{m_{\text {microporous }}}\right) * 100 \% & \text { Equation 2 }
\end{array}
$$

Where

$\varepsilon_{\text {tot }}=$ total porosity $(\%)$

$\varepsilon_{\text {macro }}=$ macroporosity $(\%)$

$\mathrm{m}_{\text {burnt }}=$ average mass sample (after burning out polymer)

(g)

$\mathrm{m}_{\text {microporous }}=$ average mass microporous sample $(\mathrm{g})$

$\mathrm{V}=$ volume sample $\left(\mathrm{cm}^{3}\right)$

$\rho_{\text {HAP }}=$ density hydroxy apatite $\left(\mathrm{g} / \mathrm{cm}^{3}\right)$

\section{Surgical procedure}

Forty healthy young-adult ( $\sim 8$ weeks old) male Wistar rats weighing $265 \pm 10 \mathrm{~g}$ were included as experimental 
animals. National guidelines for the care and use of laboratory animals were observed. The Experimental Animal Committee of the Radboud University reviewed and approved the research.

Anesthesia was induced and maintained using isoflurane inhalation (Rhodia Organique Fine Limited, Avonmouth, Bristol, England). To minimize postoperative discomfort, $0.02 \mathrm{mg} / \mathrm{kg}$ of buprenorphine (Temgesic, Reckitt Benckiser Health Care Limited, Schering-Plough, United Kingdom) was administered intraperitoneally before surgery and subcutaneously for 2 days after surgery.

After anesthesia, each rat was immobilized on its abdomen, and the skull was shaved and disinfected with chlorhexidine. A longitudinal incision was made from the nasal bone to the occipital protuberance. To minimize pain, Lidocaine HCL 1\% (B. Braun, Melsungen, Germany) was dripped at the periosteum before incision and exposure of the parietal bone. A full-thickness bone defect was created in the dorsal part of the parietal cranium central to the sagittal suture with a dental drill machine (Elcomed 100, W\&H Dentalwerk Salzburg, Austria) with a trephine burr (ACE Dental Implant System, Brockton, MA) with an outside diameter of $8.0 \mathrm{~mm}$. An earlier study showed that this diameter is critically sized after 12 weeks of implantation. ${ }^{15}$ The bone defect was drilled under continuous saline cooling and with care for the underlying dura mater and sagittal sinus. After removing the bone segment, an implant was placed. Although $\mathrm{CaP}$ cement can be injected in vivo, in the present study, we choose prehardened implants because the cranial defect model did not allow for injection because of the thin bone rim and fragile underlying dura mater. The rats were randomly assigned to one of the five groups ( $n=8$ for each implant and time period). The periosteal tissue was closed using nonresorbable Prolene 5-0 (Ethicon Inc, Somerville, NJ) suture material. The skin was closed with resorbable Vicryl 4-0 sutures (Johnson \& Johnson, St.Stevens-Woluwe, Belgium). Implantation time was 12 weeks.

Four rats in each group received in vivo fluorochromes. Tetracycline (yellow), calcein (green), and alizarin complexon (red) were administered subcutaneously $(25 \mathrm{mg} / \mathrm{kg})$, respectively 1,6 , and 11 weeks after surgery.

The animals were housed individually in cages. Intake of fluids and food was monitored in the first days postoperatively, and the animals were observed for signs of pain, infection, and proper activity. At the end of the implantation time, the rats were sacrificed using an overdose of carbon dioxide.

\section{Histology and histomorphometrical procedures}

After sacrifice, implants with surrounding tissue were retrieved, fixed in phosphate-buffered formaldehyde solution (pH 7.4) and dehydrated in increasing ethanol concentrations (70-100\%). The majority of the specimens (6/8) were embedded in methylmethacrylate (non-decalcified). Multiple thin sections $(10 \mu \mathrm{m})$ were prepared perpendicularly at different levels through the implants using a microtome with diamond blade (Leica Microsystems SP 1600, Nussloch, Germany). These slides were stained with methylene blue and basic fuchsin. At least three sections of each implant were examined with light microscopy (Leica Microsystems AG, Wetzlar, Germany). In addition, the sections were quantitatively scored using computer-based image analysis techniques (Leica Qwin Pro-image analysis system). From digitalized images (magnitude 1.6x), the amount of remained cement and newly formed bone were determined within the region of interest (a standardized area within the boundaries of the implants). As a reference for the initial $(t=0)$ quantity of cement, nonimplanted samples $(n=6)$ were scored as well. The amounts of cement and newly formed bone were expressed as a percentage of the region of interest. All data as presented are the average of the measurements of the three evaluated sections for each sample.

From the specimens of the rats that received fluorochromes in vivo, two additional unstained sections were prepared and analyzed using a reflectant fluorescence microscopy with an excitation filter of 470 to $490 \mathrm{~nm}$ (Leica Microsystems AG).

Two of the eight specimens were fixed in $10 \%$ buffered formalin ( $\mathrm{pH}$ 7.4) for 2 days, decalcified in ethylenedinitrilotetraacetic acid (EDTA, Merck KGaA, Darmstadt, Germany) for 6 days, dehydrated through graded ethanols, and embedded in paraffin. Using a microtome (Leica RM 2165, Leica Microsytems, Nussloch, Germany), thin $(6 \mu \mathrm{m})$ perpendicular sections were prepared and stained with hematoxylin and eosin. In addition, a Goldner's Masson trichrome stain was used to distinguish connective tissue and the mineralization grade of bone. Tartrate-resistant acid phosphatase (TRAP) staining was applied to determine osteoclastic activity. To perform TRAP staining, the sections were deparaffinized and preincubated in $0.2 \mathrm{M}$ TRIS-magnesium chloride $\left(\mathrm{MgCl}_{2}\right)$ buffer $(\mathrm{pH} 9.0)$ for $2 \mathrm{~h}$ at $37^{\circ} \mathrm{C}$. After rinsing with distilled water, the sections were incubated in a solution consisting of hexazotized pararosaniline $(4 \mathrm{~mL})$, naphthol ASBI phosphate $(25 \mathrm{mg}), \mathrm{N}, \mathrm{N}$-dimethylformamide $(2.5 \mathrm{~mL})$, veronal buffer $(12.5 \mathrm{~mL})$, and $\mathrm{MgCl}_{2}(0.5 \mathrm{~g})(\mathrm{pH} 5.0)$ at $37^{\circ} \mathrm{C}$. Positive staining developed within $30 \mathrm{~min}$. The sections were then counterstained for $1 \mathrm{~min}$ with hematoxylin, rinsed in water, dehydrated, and mounted with di-N-butyl phthalate in xylene (BDH Laboratory Supplies, Poole, England). Positive control sections were included.

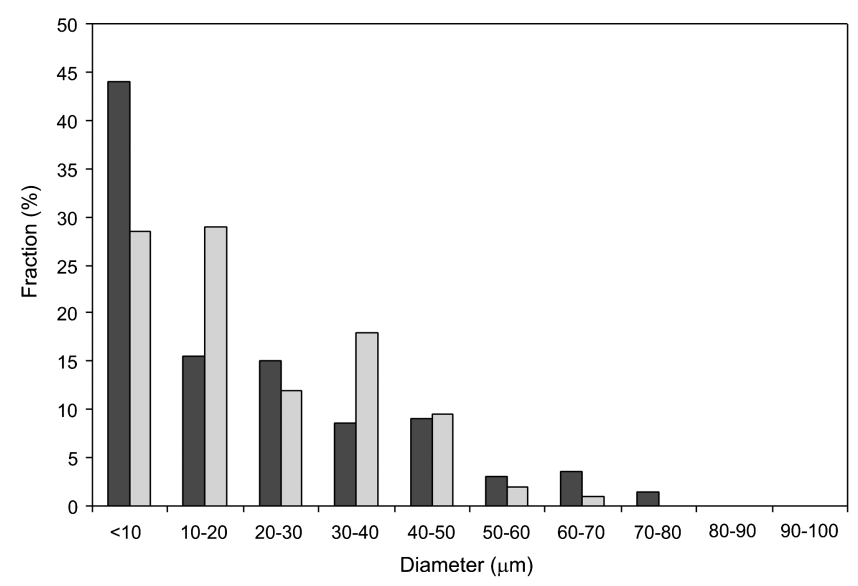

FIG. 1. Size distribution of the high-molecular-weight (HMW; black bar) and low-molecular-weight (LMW; grey bar) poly(DL-lactic-co-glycolic acid) (PLGA) microparticles prepared using a double-emulsion solvent-extraction technique (group size $=200$ ). 
FIG. 2. Scanning electron microscopy (SEM) images of (A) HMW and (B) LMW PLGA microparticles.
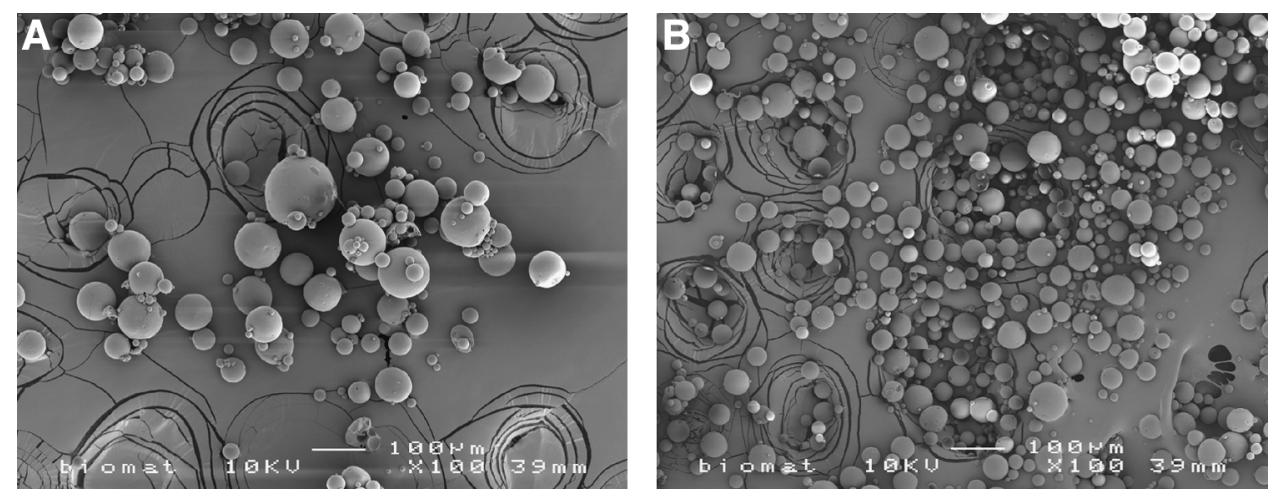

\section{Statistical analysis}

Statistical analysis was performed using GraphPad Instat version 3.05 (GraphPad Software Inc., San Diego, CA), using an unpaired $t$-test with Welch correction and a one-way analysis of variance (ANOVA). Differences were considered significant at $p$-values less than 0.05 .

\section{Results}

\section{Characterization of PLGA microparticles}

Figure 1 shows the size distribution of the HMW and LMW PLGA microparticles prepared using a double-emulsion solvent-extraction technique. The average size of the microparticles, as determined with image analysis, was $20 \pm 18 \mu \mathrm{m}$ for the HMW PLGA and $21 \pm 14 \mu \mathrm{m}$ for the LMW PLGA. SEM images revealed spherical particles with a smooth surface for the HMW (Fig. 2A) and the LMW particles (Fig. 2B).

\section{Characterization of $\mathrm{CaP}$ cement composites}

In Table 1 , the total porosity and macroporosity of the different implant formulation are shown. The 100HMW implants were significantly less porous than the other implants (ANOVA). No significant differences in porosity were seen between the other formulations. After the composites were removed from the molds, SEM images showed intact HMW PLGA microparticles incorporated into the CaP cement (Fig. 3A). The major part of the LMW PLGA microparticles, on the other hand, was more or less fused with the cement (Fig. 3B).

Table 1. Percentage of Total Porosity AND Macroporosity IN THE DifFERENT IMPLANT FORMULATIONS

\begin{tabular}{|c|c|c|}
\hline & Total porosity (\%) & Macroporosity (\%) \\
\hline & \multicolumn{2}{|c|}{ Mean \pm Standard Deviation } \\
\hline 100HMW & $65.8 \pm 0.6^{*}$ & $42.1 \pm 0.9^{*}$ \\
\hline 25LMW & $69.1 \pm 1.5$ & $47.7 \pm 2.4$ \\
\hline 50LMW & $70.1 \pm 0.5$ & $49.5 \pm 0.9$ \\
\hline 75LMW & $70.7 \pm 0.5$ & $50.4 \pm 0.8$ \\
\hline 100LMW & $69.6 \pm 1.2$ & $48.6 \pm 2.1$ \\
\hline
\end{tabular}

The $100 \%$ high-molecular-weight (HWW) poly(DL-lactic-coglycolic acid) implants (100HMW) were significantly $\left.{ }^{*}\right)$ less porous than the other groups $(p<0.001$, analysis of variance).

LMW, low molecular weight.

\section{Descriptive light microscopy}

Figure 4 shows an overview of the histological sections of the different formulations after 12 weeks of implantation. The integrity of the implants was maintained for all groups. Minimal edge degradation with rounded implant edges was seen in the 100HMW and 25LMW groups (Fig. 4A, B), where
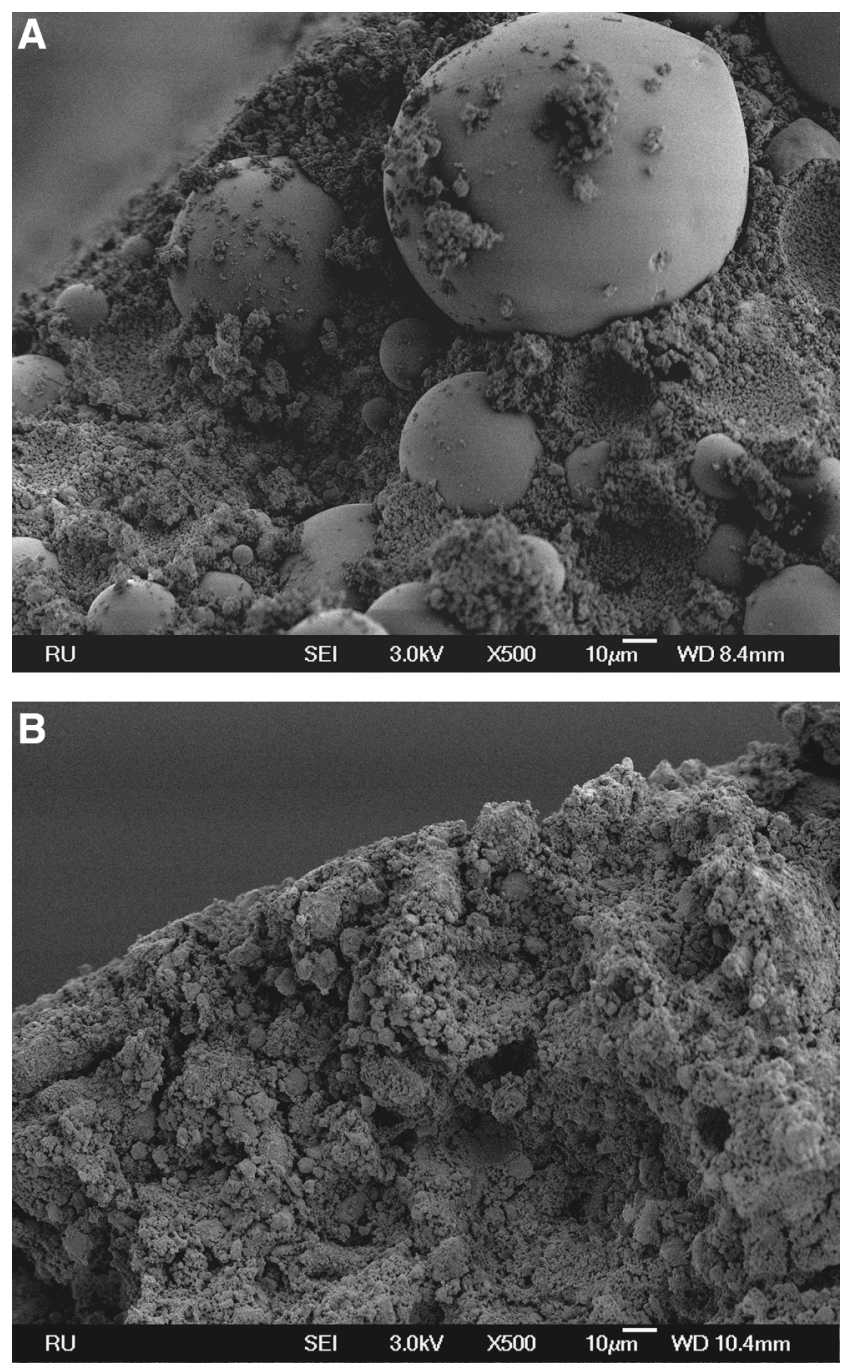

FIG. 3. SEM images of (A) 100\% HMW and (B) 100\% LMW PLGA-calcium phosphate $(\mathrm{CaP})$ cement composites before implantation. 

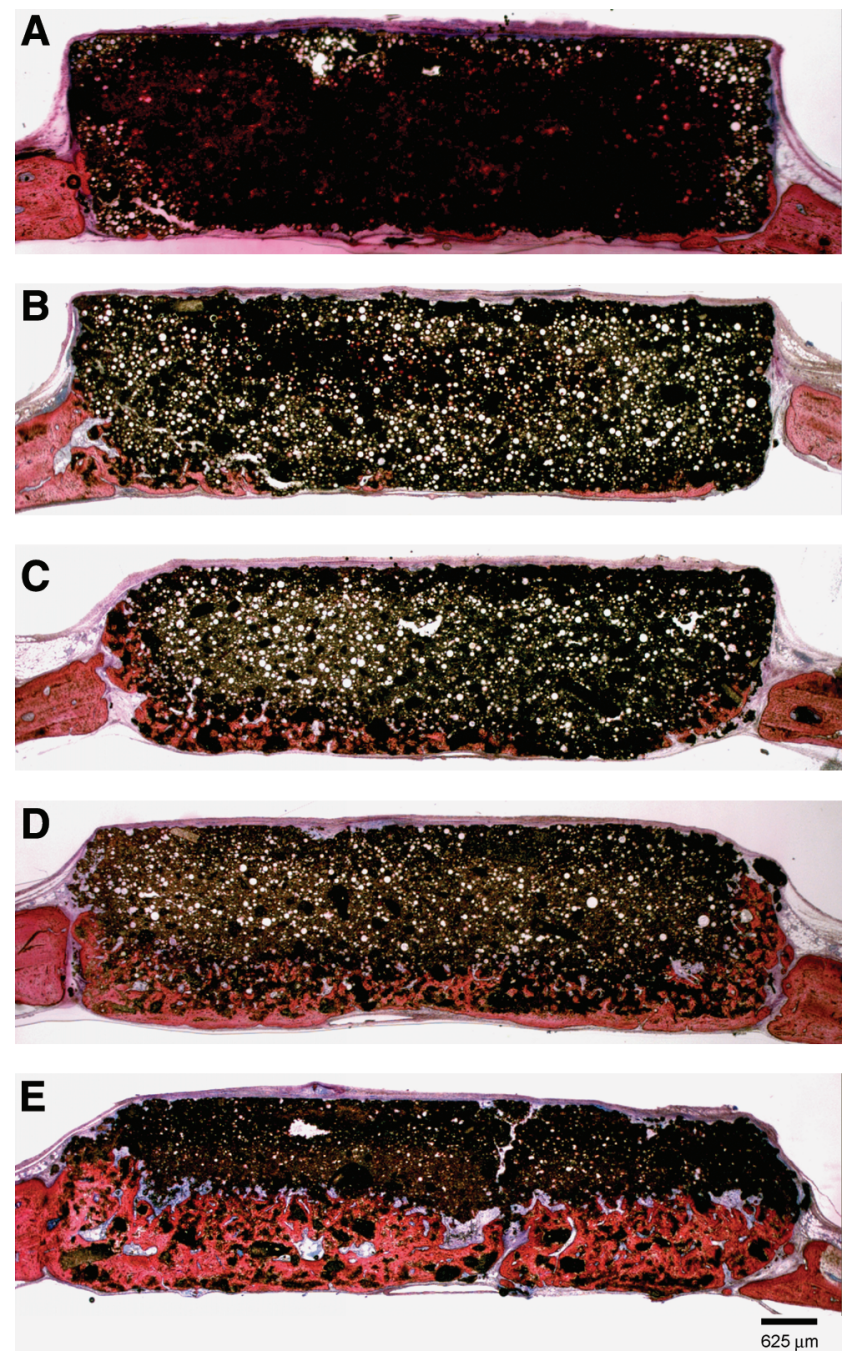

FIG. 4. Histological sections of the different formulations after 12 weeks of implantation. (A) 100\% HMW; (B) 25\% LMW, (C) 50\% LMW, (D) 75\% LMW and (E) 100\% LMW PLGA-CaP cement composites. Methylene blue and basic fuchsin staining. Color images available online at www .liebertonline.com/ten.

in the groups with $50 \%$ or more LMW PLGA microparticles (Fig. 4C-E), implant degradation had clearly occurred, with the highest degree in the 100LMW group. All formulations showed ingrowth of bone, fibrous tissue, or bone marrowlike tissue in the superficially located PLGA pores. However, in the center of the implants, cells were found only in the 75LMW and 100LMW groups (Fig. 5A) and not in the 100HMW, 25LMW, or 50LMW specimens (Fig. 5B). None of the specimens revealed signs of inflammation, as characterized by the absence of inflammatory cells.

Bone formation was seen at the defect edges and dura side of all specimens, with the greatest amount of bone in the groups with a higher percentage of LMW PLGA microparticles. Mature bone was deposited directly onto the cement surface, without an intervening fibrous tissue layer, and enclosed bone marrow-like tissue in the specimens with $50 \%$ or more LMW PLGA microparticles (Fig. 6). Bone bridging, as characterized by a bone layer covering the whole defect at
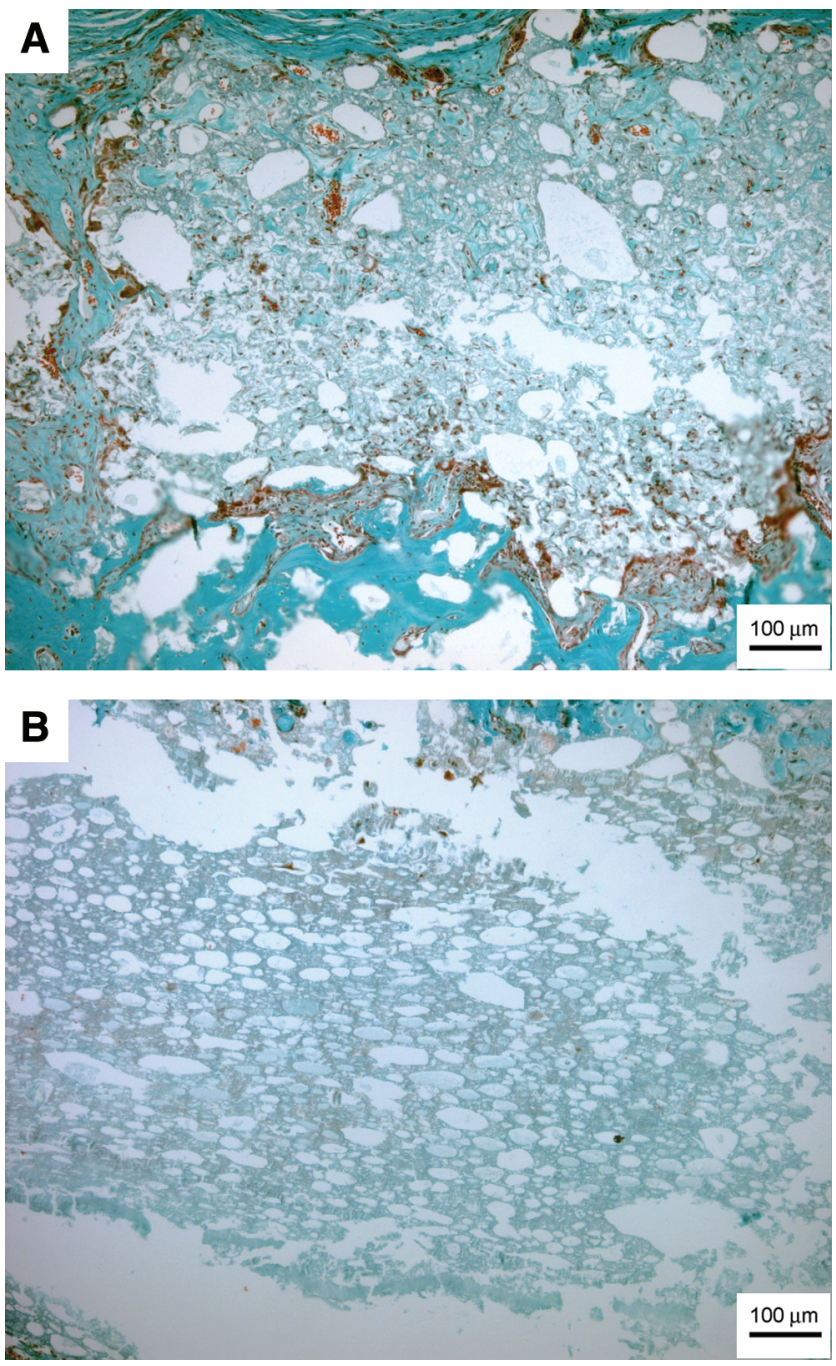

FIG. 5. Histological sections showing the center of a (A) 100\% LMW and (B) 25\% LMW implant. Goldner's Masson trichrome staining. Cellular ingrowth was not seen in the center of the $25 \%$ LMW implant. Color images available online at www.liebertonline.com/ten.

the dura side, was seen in none of the 100HMW, one 25LMW, two 50LMW, five 75LMW, and five 100LMW implants out of six implants per group (Fig. 4A-E).

TRAP staining revealed osteoclastic activity in all samples. The descriptive evaluation indicated that the TRAP staining was positive primarily in the implants with a larger amount of LMW PLGA microparticles, indicating extensive active bone remodeling in these specimens (Fig. 7).

Fluorescence microscopy confirmed that bone formation started at the dura side, because green bands were detected here (calcein administered 6 weeks postoperatively) and then continued toward the periosteum (red bands of alizarin complexon administered 11 weeks after surgery) (Fig. 8). Yellow tetracycline bands (1 week postoperatively) were found mainly at the defect edges.

\section{Histomorphometry}

The histomorphometrical results are depicted in Table 2. All implant types showed significant degradation after 12 


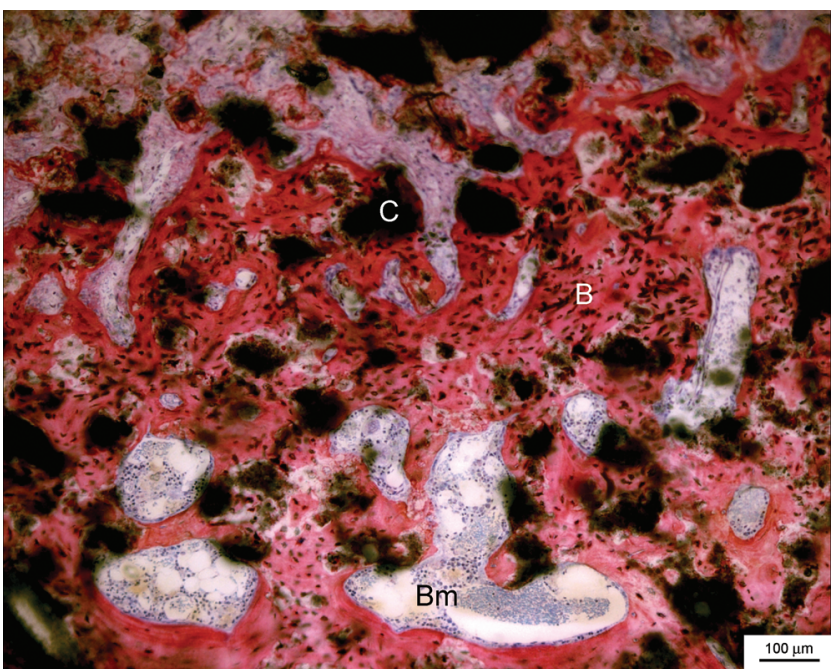

FIG. 6. Mature bone (B) with bone marrow-like tissue (Bm) was deposited directly onto the cement surface (C) of a $100 \%$ LMW implant. Methylene blue and basic fuchsin staining. Color images available online at www.liebertonline.com/ten.

weeks of implantation from the initial $(t=0)$ amount of cement (unpaired $t$-test with Welch correction, $p<0.01$ ). Comparing the relative decreases $(\Delta \mathrm{T} 0 / 12)$ in amount of implant after 12 weeks of implantation of the different formulations, it appeared that 100HWW and 25LMW were similar and were significantly less degraded than the similar groups of 50LMW and 75LMW (unpaired $t$-test with Welch correction, $p<0.01)$. In addition, the relative decrease of the 100LMW implants after 12 weeks $(40 \pm 6 \%)$ was significantly greater than for all other formulations (unpaired $t$-test with Welch correction, $p<0.003$ ).

The percentage of new bone formation was comparable between the 100HMW and 25LMW groups. Also, bone formation in the 50LMW group was not significantly different from the 100HMW and 25LMW formulations (unpaired $t$-test with Welch correction, $p=0.08$ and $p=0.06$, respectively). Bone formation in the 75LMW implants was significantly greater than in these groups $(p<0.04)$. However, the 100LMW showed the significantly greatest amount of newly formed bone $(26 \pm 6 \%)$ (unpaired $t$-test with Welch correction, $p<0.02)$.

\section{Discussion}

$\mathrm{CaP}$ cements are promising materials that can be used in diverse targeted bone-related treatments, but their degradative behavior is a property that has to be optimized. ${ }^{7}$ In the present study, PLGA microparticles were incorporated into CaP cement with diverse proportions of LMW and HMW PLGA to investigate the influence of molecular weight on bioactive behavior. A higher percentage of LMW PLGA microparticles in the composites was associated with a better bone biological response. In the current study, no control materials without addition of PLGA were included. Extensive previous animal testing had proved that the degradation of nonporous $\mathrm{CaP}$ cement is limited. ${ }^{20-22}$

Composites of PLGA and CaP cement have been shown to be biocompatible and to form macroporous scaffolds after
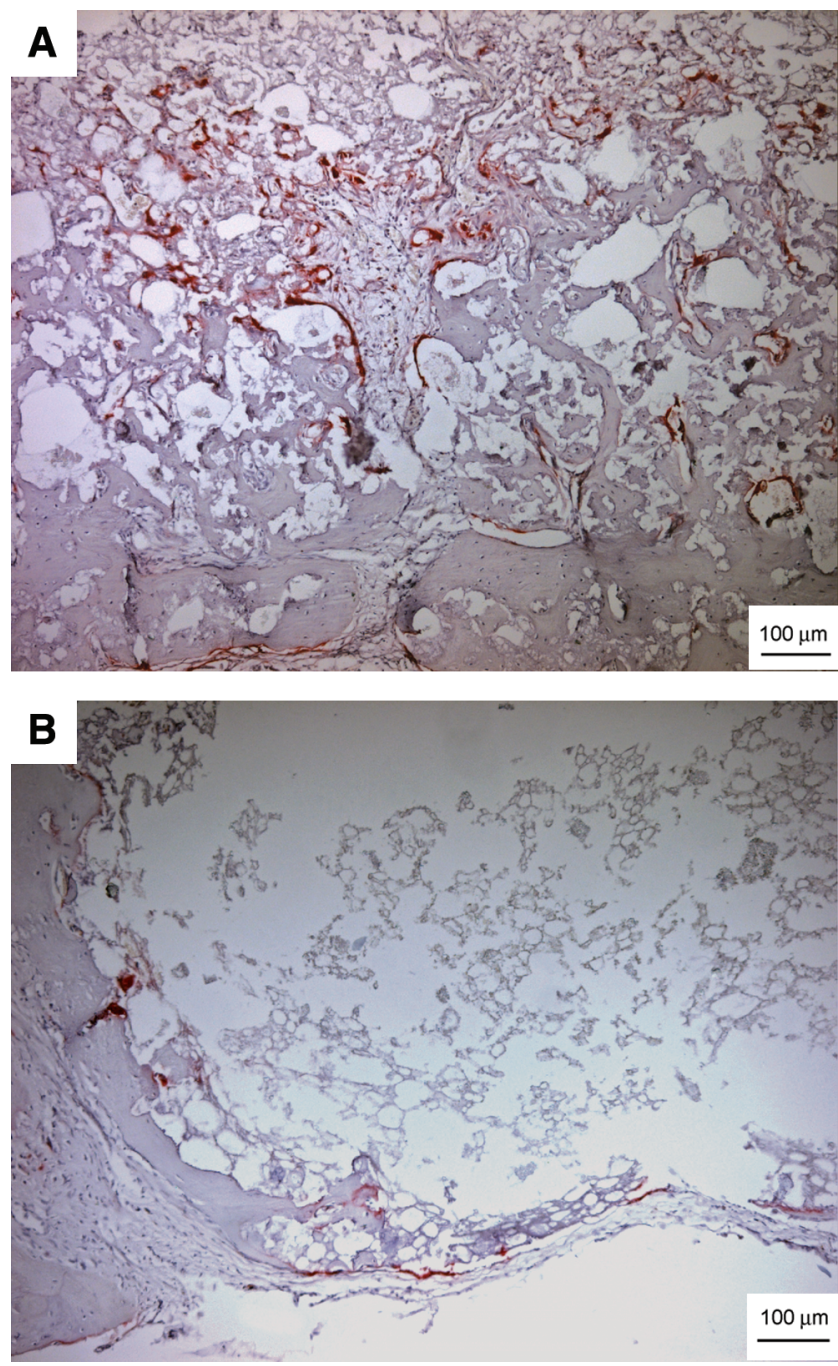

FIG. 7. Tartrate-resistant acid phosphatase-positive cells in (A) 100\% LMW and (B) 100\% HMW implants. Color images available online at www.liebertonline.com/ten.

polymer degradation in previous in vitro studies or in noncritical-sized animal models. ${ }^{10,12,23-25}$ Also, in the present study, using a critical-sized defect model, all formulations of PLGA and CaP cement showed biocompatibility and some bone ingrowth into the PLGA pores. Nevertheless, differences were seen between the formulations, with a higher amount of LMW PLGA resulting in better implant degradation and bone ingrowth. PLGA is a synthetic polymer that degrades via a random hydrolytic mechanism that is dependent on several factors, including molecular weight. ${ }^{16,26}$ Low molecular weight PLGA degrades faster than high molecular weight PLGA. ${ }^{16,27}$ Habraken et al. ${ }^{11}$ determined the $\mathrm{M}_{\mathrm{w}}$ loss of PLGA microparticles with a $\mathrm{M}_{\mathrm{w}}$ similar to that of our HMW group $(48.0 \pm 1.6 \mathrm{kDa})$ inside CaP cement samples when incubated in phosphate buffered saline at $37^{\circ} \mathrm{C}$. They found that a linear decrease with approximately 20\% PLGA remained after 6 weeks and complete degradation after 12 weeks of incubation. In contrast, LMW PLGA microparticles $(4.54 \pm 0.03 \mathrm{kDa})$ showed a $\mathrm{M}_{\mathrm{w}}$ loss of approximately $50 \%$ after 4 weeks and complete degradation after 6 weeks (unpublished data). As in the present study, no 


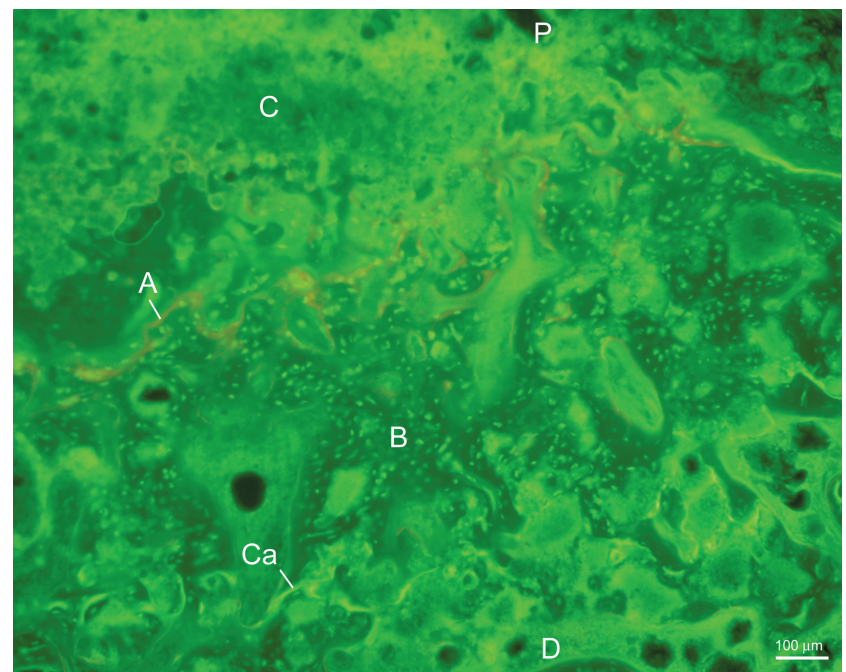

FIG. 8. Fluorescence microscopic image of a $100 \%$ LMW implant (C). Ca, calcein green administered 6 weeks after surgery; A, alizarin complexon red administered 11 weeks after surgery; $\mathrm{P}$, periosteal side of the implant; $\mathrm{D}$, dural side of the implant; $\mathrm{B}$, bone. Color images available online at www.liebertonline.com/ten.

significant difference was seen in microparticle size between HMW and LMW PLGA or in porosity between the 25LMW, 50LMW, 75LMW, and 100LMW samples; this different rate of PLGA degradation most probably explains the differences noticed in implant degradation and bone ingrowth between the groups. Evidently, a high percentage of fast-degrading LMW PLGA in the CaP cement composites facilitated the formation of a macroporous structure and thereby the degradation of the implant, followed by bone ingrowth. Considering the observed degradation of the 75LMW and 100LMW samples and the lack of PLGA degradation and tissue ingrowth into the other materials, there seems to be no direct correlation between the originally "designed" material porosity (by the inclusion of PLGA microparticles) and final bone ingrowth. Apparently, the driving factor for bone ingrowth is PLGA degradation. Degradation appeared to be associated with osteoclast activity, because TRAP-positive cells were detected in all formulations but especially in the high LMW (75 and 100 LMW) groups. Bone formation started at the defect edges and dura side, as displayed with fluorescent labeling, and then continued toward the periosteal side, which is consistent with preceding investigations ${ }^{28,29}$ and is probably explained by the presence of preosteoblastic cells in the dura mater. ${ }^{28}$ The rate of degradation and bone ingrowth were similar, because the integrity of the scaffolds was maintained. For future studies, it would be interesting to perform mechanical tests to determine the shear strength of the implants.

SEM images generated of the composites before implantation revealed an important difference in structure between the formulations. Although the HMW microparticles showed the same morphology in the cement as before the mixing procedure, the LMW microparticles lost their spherical shape, and the majority were fused with the $\mathrm{CaP}$ cement. This observation can explain why the percentage of implant seems to be LMW concentration dependant at T0 (Table 2). Furthermore, this finding might indicate a different interconnectivity between the groups (i.e., the merging of the LMW PLGA microparticles within the CaP cement improved the interconnection between the particles). The possession of a network of linked pores is an important criterion for biomaterials because it enables cellular and vascular penetration, and bone ingrowth inside scaffolds is thereby favored. ${ }^{30}$ Interconnectivity between spheres in a solid matrix is supposed to be present when the volume fraction exceeds $0.4 .^{31}$ The 100HMW group just surpassed this limit, with $42 \%$ macroporosity; all the other formulations possessed a macroporosity or approximately $50 \%$ and should have had an interconnective system confirm this hypothesis. Nevertheless, only the 75LMW and 100LMW formulations showed cellular ingrowth throughout the sample, indicating that the particles were interconnected. The 25LMW and $50 \mathrm{LMW}$ groups might possess an interconnective system, but because of the poor degradation of the HMW PLGA microspheres within 12 weeks of implantation, this could not be detected in the present study.

As stated above, differences between the 100HMW and 100LMW groups were seen in cellular entry in the middle of the implant. Tissue ingrowth throughout the implants as seen in the study of Ruhé et al. ${ }^{12}$ where non-critical sized defects were created, was only noticed in the 75LMW and 100LMW samples. Apparently, the microparticles in the groups containing less LMW PLGA were entrapped in the center of the implant and thereby withdrawn from necessary

Table 2. Percentage of Implant and New Bone in the Region of Interest as Determined with Histomorphometry

\begin{tabular}{|c|c|c|c|c|}
\hline & \multicolumn{3}{|c|}{$\%$ Implant } & \multirow[b]{3}{*}{ \% Bone } \\
\hline & $\begin{array}{c}\text { Before } \\
\text { Implantation }\end{array}$ & $\begin{array}{l}12 \text { Weeks After } \\
\text { Implantation }\end{array}$ & $\begin{array}{l}\text { Difference in \% of } \\
\text { Implant Between the } \\
\text { Two Time Points }\end{array}$ & \\
\hline & \multicolumn{3}{|c|}{ Mean \pm Standard Deviation } & \\
\hline $100 \mathrm{HMW}$ & $84 \pm 3$ & $74 \pm 3$ & $10 \pm 3$ & $8 \pm 2$ \\
\hline 25 LMW & $81 \pm 2$ & $72 \pm 4$ & $8 \pm 4$ & $9 \pm 1$ \\
\hline $50 \mathrm{LMW}$ & $86 \pm 4$ & $65 \pm 3$ & $21 \pm 3$ & $11 \pm 2$ \\
\hline 75 LMW & $86 \pm 3$ & $65 \pm 8$ & $21 \pm 8$ & $17 \pm 5$ \\
\hline 100 LMW & $90 \pm 2$ & $50 \pm 6$ & $40 \pm 6$ & $26 \pm 6$ \\
\hline
\end{tabular}

$\mathrm{T}=0$ : before implantation; $\mathrm{t}=12$ weeks: after 12 weeks implantation; $\Delta \mathrm{T} 0 / 12$ : difference in $\%$ of implant between the two time points. HMW, high molecular weight; LMW, low molecular weight. 
fluids for degradation. This entrapment might also cause problems at a later time point, as described by Ignatius et al., ${ }^{32}$ who implanted composites of PLA $\left(\mathrm{M}_{\mathrm{w}} 91 \mathrm{kDa}\right)$ and alpha-tocopherol in tibial bone defects of sheep for 6, 12, and 24 months. After 6 and 12 months of implantation, the implants were filled with newly formed bone, and only a mild inflammatory response was observed, but after 24 months, a strong inflammatory reaction was seen, and the newly formed bone was partly osteolytic. It was suggested that newly formed bone had encapsulated the acid degradation products of PLA, which degraded at this later time point and caused the adverse reaction. A better coordination of the degradation times of the polymer and ceramic component was recommended. The coordination in degradation seemed to be effective in our predominantly LMW PLGA composites, with PLGA degradation in these scaffolds occurring at an early moment when no capsule was present and clearing of the acid products was not prevented, resulting in healing of the critical-sized bone defects.

For all formulations, the dimension of the microparticles and thereby the pore size of the scaffolds was smaller than in previous studies of osteoconductive biomaterials. In general, it is said that a pore size of at least 50 to $100 \mu \mathrm{m}$ is necessary to allow bone formation, ${ }^{33,34}$ although the narrow pore size of $21 \pm 14 \mu \mathrm{m}$ appeared to be large enough in our LMW PLGA group to obtain cellular entry and bone ingrowth throughout the implants. Lu et al. ${ }^{30}$ stated that, in addition to pore size, the density of interconnections in porous bioceramics plays an important role for bone formation, and this seems also to be true of our LMW implants.

The finding that composites of CaP cement and LMW PLGA microparticles are able to bridge critical-sized cranial defects without the inclusion of growth factors is most important. The amount of bone ingrowth in the LMW samples $(26 \pm 6 \%)$ was considerably higher than in HMW implants, including recombinant human bone morphogenetic protein 2 $(16 \pm 2 \%)$, as we found recently using exactly the same animal model and implantation time. ${ }^{15}$ So far, we know of no previous studies that were able to show that critical-sized defects could be healed with a material containing no growth factors. This is important because growth factors are costly and there are significant problems with the bioavailability of these compounds when included in CaP materials. ${ }^{15}$ Whether the combination of LMW PLGA with growth factors has even superior bone biological behavior deserves further investigation.

\section{Conclusion}

In conclusion, the molecular weight of PLGA in CaP cement composites influences implant degradation and new bone formation. A higher percentage of fast-degrading LMW PLGA microparticles increases the biological activity of the composites more than HMW PLGA microparticles. The larger interconnectivity in the LMW samples probably explains this difference. Composites with 100\% LMW PLGA microparticles are capable of bridging critical-sized cranial defects without the inclusion of growth factors.

\section{Acknowledgments}

The authors would like to thank Natasja van Dijk for her assistence with the histological preparations.

\section{Disclosure Statement}

The authors have no competing financial interests.

\section{References}

1. Kenny, S.M., Buggy, M. Bone cements and fillers: a review. J Mater Sci Mater Med 14, 923, 2003.

2. Heini, P.F., Berlemann, U. Bone substitutes in vertebroplasty. Eur Spine J 10 Suppl 2, 205, 2001.

3. Larsson, S., Bauer, T.W. Use of injectable calcium phosphate cement for fracture fixation: a review. Clin Orthop Relat Res 23, 2002.

4. LeGeros, R.Z. Properties of osteoconductive biomaterials: calcium phosphates. Clin Orthop 81, 2002.

5. Ruhé, P.Q., Wolke, J.G.C., Spauwen, P.H.M., Jansen, J.A. Calcium phosphate ceramics for bone tissue engineering. In: Fisher, J.P., Mikos, A.G., Brozino, A.D., eds., Tissue Engineering. CRC Press, Boca Raton, FL, 2007, pp. 9/1-9/18.

6. Jansen, J., Ooms, E., Verdonschot, N., Wolke, J. Injectable calcium phosphate cement for bone repair and implant fixation. Orthop Clin North Am 36, 89, 2005.

7. Ambard, A.J., Mueninghoff, L. Calcium phosphate cement: review of mechanical and biological properties. J Prosthodont 15, 321, 2006.

8. Ooms, E.M., Wolke, J.G., van der Waerden, J.P., Jansen, J.A. Trabecular bone response to injectable calcium phosphate (Ca-P) cement. J Biomed Mater Res 61, 9, 2002.

9. Del Real, R.P., Ooms, E., Wolke, J.G., Vallet-Regi, M., Jansen, J.A. In vivo bone response to porous calcium phosphate cement. J Biomed Mater Res 65A, 30, 2003.

10. Simon, C.G. Jr, Khatri, C.A., Wight, S.A., Wang, F.W. Preliminary report on the biocompatibility of a moldable, resorbable, composite bone graft consisting of calcium phosphate cement and poly(lactide-co-glycolide) microspheres. J Orthop Res 20, 473, 2002.

11. Habraken, W.J., Wolke, J.G., Mikos, A.G., Jansen, J.A. Injectable PLGA microsphere/calcium phosphate cements: physical properties and degradation characteristics. J Biomater Sci Polymer Edn 17, 1057, 2006.

12. Ruhe, P.Q., Hedberg, E.L., Padron, N.T., Spauwen, P.H., Jansen, J.A., Mikos, A.G. Biocompatibility and degradation of poly(DL-lactic-co-glycolic acid)/calcium phosphate cement composites. J Biomed Mater Res A 74, 533, 2005.

13. Ruhe, P.Q., Hedberg, E.L., Padron, N.T., Spauwen, P.H., Jansen, J.A., Mikos, A.G. rhBMP-2 release from injectable poly(DL-lactic-co-glycolic acid)/calcium-phosphate cement composites. J Bone Joint Surg Am 85-A Suppl 3, 75, 2003.

14. Ruhe, P.Q., Boerman, O.C., Russel, F.G., Spauwen, P.H., Mikos, A.G., Jansen, J.A. Controlled release of rhBMP-2 loaded poly(dl-lactic-co-glycolic acid)/calcium phosphate cement composites in vivo. J Control Release 106, 162, 2005.

15. Bodde, E.W., Boerman, O.C., Russel, F.G., Mikos, A.G., Spauwen, P.H., Jansen, J.A. The kinetic and biological activity of different loaded rhBMP-2 calcium phosphate cement implants in rats. J Biomed Mater Res A 87, 780, 2008.

16. Shive, M.S., Anderson, J.M. Biodegradation and biocompatibility of PLA and PLGA microspheres. Adv Drug Deliv Rev 28, 5, 1997.

17. Lu, L., Stamatas, G.N., Mikos, A.G. Controlled release of transforming growth factor beta1 from biodegradable polymer microparticles. J Biomed Mater Res 50, 440, 2000.

18. Peter, S.J., Lu, L., Kim, D.J., Stamatas, G.N., Miller, M.J., Yaszemski, M.J., Mikos, A.G. Effects of transforming growth factor beta1 released from biodegradable polymer micro- 
particles on marrow stromal osteoblasts cultured on poly(propylene fumarate) substrates. J Biomed Mater Res 50, $452,2000$.

19. Cleek, R.L., Rege, A.A., Denner, L.A., Eskin, S.G., Mikos, A.G. Inhibition of smooth muscle cell growth in vitro by an antisense oligodeoxynucleotide released from poly(DLlactic-co-glycolic acid) microparticles. J Biomed Mater Res 35, 525, 1997.

20. Wolke, J.G., Wenz, R., Ooms, E.M., Boltong, M.G., Driessens, F.C., Jansen, J.A. Physicochemical properties, composition and resorption behavior in vivo of high-resistant calcium phosphate cements. Rev Chir Orthop Reparatrice Appar Mot 86, 511, 2000.

21. Comuzzi, L., Oooms, E., Jansen, J.A. Injectable calcium phosphate cement as a filler for bone defects around oral implants: an experimental study in goats. Clin Oral Impl Res 13, 304, 2002.

22. Oooms, E.M., Wolke, J.G., van den Heuvel, M.T., Jansen, J.A. Histological evaluation of the bone response to calcium phosphate cement implanted in cortical bone. Biomaterials 24, 989, 2003.

23. Link, D.P., van den Dolder, J., Jurgens, W.J., Wolke, J.G., Jansen, J.A. Mechanical evaluation of implanted calcium phosphate cement incorporated with PLGA microparticles. Biomaterials 27, 4941, 2006.

24. Ruhe, P.Q., Hedberg-Dirk, E.L., Padron, N.T., Spauwen, P.H., Jansen, J.A., Mikos, A.G. Porous poly(DL-lactic-coglycolic acid)/calcium phosphate cement composite for reconstruction of bone defects. Tissue Eng 12, 789, 2006.

25. Dagang, G., Haoliang, S., Kewei, X., Yong, H. Long-term variations in mechanical properties and in vivo degradability of CPC/PLGA composite. J Biomed Mater Res B Appl Biomater 82, 533, 2007.

26. Panyam, J., Dali, M.M., Sahoo, S.K., Ma, W., Chakravarthi, S.S., Amidon, G.L., Levy, R.J., Labhasetwar, V. Polymer degradation and in vitro release of a model protein from poly(D,L-lactide-co-glycolide) nano- and microparticles. J Control Release 92, 173, 2003.

27. Tracy, M.A., Ward, K.L., Firouzabadian, L., Wang, Y., Dong, N., Qian, R., Zhang, Y. Factors affecting the degradation rate of poly(lactide-co-glycolide) microspheres in vivo and in vitro. Biomaterials 20, 1057, 1999.

28. Wang, J., Glimcher, M.J. Characterization of matrix-induced osteogenesis in rat calvarial bone defects: II. Origins of boneforming cells. Calcif Tissue Int 65, 486, 1999.

29. Takagi, K., Urist, M.R. The reaction of the dura to bone morphogenetic protein (bmp) in repair of skull defects. Ann Surg 196, 100, 1982.

30. Lu, J.X., Flautre, B., Anselme, K., Hardouin, P., Gallur, A., Descamps, M., Thierry, B. Role of interconnections in porous bioceramics on bone recolonization in vitro and in vivo. J Mater Sci Mater Med 10, 111, 1999.

31. Gurland, J. An estimate of contact and continuity of dispersion in opaque samples. Trans Met Soc AIME 236, 642, 1966.

32. Ignatius, A.A., Betz, O., Augat, P., Claes, L.E. In vivo investigations on composites made of resorbable ceramics and poly(lactide) used as bone graft substitutes. J Biomed Mater Res 58, 701, 2001.

33. Itala, A.I., Ylanen, H.O., Ekholm, C., Karlsson, K.H., Aro, H.T. Pore diameter of more than 100 microm is not requisite for bone ingrowth in rabbits. J Biomed Mater Res 58, 679, 2001.

34. Bobyn, J.D., Pilliar, R.M., Cameron, H.U., Weatherly, G.C. The optimum pore size for the fixation of porous-surfaced metal implants by the ingrowth of bone. Clin Orthop Relat Res 263, 1980.

Address correspondence to: John A. Jansen, D.D.S., Ph.D. Department of Periodontology and Biomaterials Radboud University Nijmegen Medical Center P.O. Box 9101,6500 HB Nijmegen The Netherlands

E-mail: j.jansen@dent.umcn.nl

Received: December 22, 2008 Accepted: April 10, 2009

Online Publication Date: May 25, 2009 
